Aims. Recent MHRA guidelines, state that Valproate medicines must no longer be used in women or girls of childbearing potential due to its highly teratogenic effects unless a Pregnancy Prevention Programme is in place. We carried out a service evaluation to determine if there was any way of identifying such patients with the aim of setting one up if required.

Background. Valproate is highly teratogenic and evidence supports that use in pregnancy leads to physical birth defects in 10 in every 100 babies (compared with a background rate of 2 to 3 in 100) and neurodevelopmental disorders in approximately 30 to 40 in every 100 children born to mothers taking Valproate. Data from a previous inpatient audit identified 35 females of childbearing age and none was on a pregnancy prevention plan. Audits done thereafter confirmed there was no system available for identifying such patients. Availability and accessibility to a synchronised IT system, which alerts when the yearly review is due is consistently identified as a contributory factor.

Method. A request via the Medicines management team was sent to the GP surgeries within the catchment area to assist in identifying female patients on their records on Valproate registered on the Pregnancy Prevention Programme.

The Plan-Do-Study-Act (PDSA) Quality Improvement (QI) model was used to bring about change. The target set to achieve was a $50 \%$ reduction in the prescriptions of Valproate in such patient groups.

Result. We had 10 out of the 50 GP surgeries contacted responded with a list of female patients on Valproate, a total of 25 patients' altogether. In total, 4 patients out of the 25 were registered on the Pregnancy Prevention Programme and the overall non-compliance rate was $86 \%$. . Factors believed to contribute to the low numbers include a lack of a system for registering women of childbearing age on the pregnancy protection plan and the recent introduction of GDPR regulation.

Conclusion. There are ongoing discussions with various stakeholders like the Medicines management team, Pharmacists, electronic records team (IT) and other clinicians regarding inserting an alert in the electronic system that reminds clinicians to register all such women on the Pregnancy Prevention Programme, while automatically creating a yearly reminder for completion of the annual risk acknowledgement form.

\section{Improving knowledge and confidence in the acute management of eating disorders and resulting complications}

Sarah Fynes-Clinton*, Clare Price, Louisa Beckford, Maisha Shahjahan and Brendan McKeown

Coventry and Warwickshire Partnership Trust ${ }^{\star}$ Corresponding author.

doi: 10.1192/bjo.2021.503

Aims. This project aimed to improve the knowledge and confidence of doctors at all levels when managing patients with eating disorders while on call.

Background. A recent survey found just $1 \%$ of doctors have the opportunity for clinical experience on eating disorders. Anecdotally, a number of junior doctors within our trust had mentioned that they felt unsure when asked to manage patients with eating disorders during their out of hours shifts.

Method. This project aimed to ascertain levels of confidence with managing patients with eating disorders, and to collect suggestions to improve this. This was achieved using a survey sent out to 97 doctors working in a Mental Health Trust.
We then utilised two of the suggestions to improve the identified areas of concern. The first method involved direct lectures. This was followed up with the creation of a poster highlighting the pertinent information which was displayed in key clinical areas. The second avenue was the creation of an information booklet covering key clinical information that is available to all on call doctors.

Result. The response rate for the survey was $37.11 \%$. The survey found that doctors lacked confidence in the management of common conditions that arise in patients admitted with eating disorders. Refeeding syndrome was identified as the greatest area of concern by responding doctors.

To assess the impact of the lectures, MCQs were given out before and after the presentation. The results were compared, and showed a clear improvement in overall knowledge, with results going from an average score of $56.6 \%$ to $80 \%$.

Conclusion. By using multiple methods to improve doctors confidence, (lectures, written information and visual posters), this quality improvement project achieved its aims in improving doctors knowledge, and through having easy access to important information, will have long term positive effects on patient care.

Evaluation of tobacco use and willingness to accept nicotine replacement therapy during stay in an acute inpatient psychiatric ward

Isabel Ganhao*, Miguel Trigo and Afonso Paixao

Centro Hospitalar Psiquiatrico de Lisboa

${ }^{*}$ Corresponding author.

doi: $10.1192 /$ bjo.2021.504

Aims. To evaluate tobacco smoking and willingness to try and to accept prescription of nicotine replacement therapy during psychiatric acute inpatient stay. When free to choose and use as desired (without imposing smoking cessation) patients may be open to nicotine replacement therapy.

Background. Tobacco smoking interventions are increasingly in demand especially for difficult to treat patient populations, as are those with severe mental illness. Implementing totally smokefree psychiatric inpatient units is challenging. Imposing smoking cessation and use of nicotine substitutes may or not be the best of strategies for smoking reduction and cessation in the mid and long term. Allowing the patient free choice as to trying and learning to use nicotine replacement therapy with supervision may constitute a more acceptable approach.

Method. From 1/1/2020 to 28/1/2020 (four weeks), 40 of the 44 patients in a general adult psychiatric inpatient unit (4 patients were too sedated or too agitated to be evaluated), with smoking restricted to a designated area and only during the day, were briefly evaluated as to their tobacco smoking habits (cigarettes/ day) and willingness to accept nicotine lozenges and patches and were invited to participate in a smoking cessation programme during or after discharge.

Result. Of the 40 patients evaluated, 26 were male and 14 were female, average age was 34 years (age range from 19 to 79 years). Diagnostic hypotheses for patients at admission were: schizophrenic disorder/schizoaffective disorder 9, bipolar disorder 7 , psychosis not otherwise specified 19, depressive disorder 1 and other $4.20(50 \%)$ were current smokers and $20(50 \%)$ were non or ex-smokers. The smokers reported smoking an average of 14 cigarettes/day. Only one patient refused to try nicotine lozenges and all other smokers accepted regular prescription of nicotine lozenges during inpatient stay. 5 patients (25\% of smokers) asked for or accepted suggestion to try nicotine patches in 
combination therapy with nicotine lozenges with the goal of smoking cessation.

Conclusion. Patients were open to a brief informal intervention targeting smoking behaviours and readily accepted trying nicotine lozenges and prescription during their inpatient stay. The regular use of nicotine replacement therapy by some patients encouraged other patients to try and accept therapy. In addition to the habitual tobacco sharing among patients, nicotine lozenges were also shared especially with newly admitted patients. The evaluation of the impact of this intervention will require a much longer period of time of implementation.

\section{"The path is smooth that leadeth on to danger": caffeine and psychosis}

Isabel Ganhao*, Goncalo Marinho, Afonso Paixa and Miguel Trigo

Centro Hospitalar Psiquiatrico de Lisboa

${ }^{*}$ Corresponding author.

doi: 10.1192/bjo.2021.505

Aims. To review literature on the importance of caffeine intake with regard to psychosis. The need for intervention with regard to caffeine intake hinges on effectively recognizing potential risks. Background. Caffeine is the most widely consumed psychoactive substance worldwide and as such is generally considered acceptable but as a competitive adenosine antagonist, it affects dopamine transmission. Patients with serious mental illness are known to have higher caffeine intakes than the general population. The hierarchy of needs for this patient population is complex, frequently leaving the intake of caffeine under the radar of clinical priorities.

Method. PubMed and Google Scholar search for caffeine/coffee and psychosis/schizophrenia

Result. Of the 43 articles that were considered relevant for clinical practice, caffeine consumption was associated with 1) appearance of psychotic symptoms and episodes (caffeine-induced psychosis) and chronic psychosis in high intake 2) exacerbation of psychosis in schizophrenic patients even in lower intakes, 3) treatment resistance possibly due to interference with antipsychotics (ex. clozapine), 4) abuse and addiction, 5) comorbidity with tobacco smoking and other addictions. Caffeine in low doses was associated with ameliorating cognitive and extrapyramidal side-effects of medication and as a potential treatment strategy for treatment-resistant schizophrenia.

Conclusion. Caffeine consumption may have a greater impact on psychotic symptoms and episodes than is recognized with negative effects outweighing any potential benefits. Greater awareness of the necessity to quantify caffeine intake and implementation of interventions to curb intake may contribute to better quality of care of serious mental illness. Further research is warranted.

\section{Monitoring lithium therapy in a CMHRS during the COVID-19 pandemic}

Katherine Gardner* and Charles Shuttleworth

Surrey and Borders Partnership NHS Foundation Trust

${ }^{*}$ Corresponding author.

doi: 10.1192/bjo.2021.506

Aims. The COVID-19 pandemic has presented a challenge in ensuring that routine monitoring can still be offered and occur in a safe and effective manner. Our aim was to continue the regular physical health monitoring of North East Hampshire CMHRS patients who are prescribed lithium during the COVID-19 pandemic, and to achieve above 90\% compliance for the monitoring standards. Lithium monitoring clinics have been established in our CMHRS since 2017, Lithium is a high-risk medication as recognised by a National Patient Safety Alert in 2009. Previous annual POMH-UK audits have identified suboptimal monitoring of Lithium patients at local and national levels.

Method. The 'Plan-Do-Study-Act' (PDSA) approach was utilised and a 'QI Bundle' formed. A database of patients who are prescribed lithium has been created and maintained. Synchronised standardised reminder letters are sent to the patients twice yearly. NICE recommends that patients on lithium have their lithium levels checked every 3 months for the first year and then at least every 6 months, plus TFTs, U\&Es, calcium and weight every 6 months (or more frequently if impaired). The Specialist Pharmacist Service advise that during the COVID-19 pandemic, if patients are not in the at-risk category then monitoring intervals can be extended by up to 3 months, but that patients in the at-risk category should have their normal monitoring intervals continued.

Lithium clinics have been held every April and October since 2017 by the Junior Doctors allocated to NE Hampshire CMHRS. This year they were conducted via telephone appointment or face to face where safe to do so with clinician in full PPE. An audit was subsequently carried out in December 2020 to assess our patient's compliance with the aforementioned NICE recommendations for lithium monitoring, the results of which were compared to previous annual audits.

Result. Seventeen patients were currently prescribed lithium within the NE Hampshire CMHRS. Over 90\% compliance with monitoring was achieved in December 2020 apart from the checking of calcium levels which was slightly below target at $82 \%$, and weight which was at $88 \%$. There was no significant reduction in monitoring standards obtained compared to data from the previous three years.

Conclusion. Routine monitoring for our patients who are prescribed Lithium was effectively and safely continued during the pandemic. Above 90\% compliance with lithium monitoring standards was nearly obtained across all areas. We will continue to offer lithium monitoring twice yearly.

Improving training and support by improving our out of hours handover, Central and North West London NHS Foundation Trust

\author{
Mehmet $\mathrm{Gez}^{*}$ and Guang Xu \\ Central and North West London NHS Foundation Trust \\ ${ }^{\star}$ Corresponding author.
}

doi: $10.1192 /$ bjo.2021.507

Aims. During out of hour handovers at St Charles Hospital - the two duty SHO (senior house officers) cover on site, whereas the on-call registrar and consultant are available to contact by phone. Some trainees may experience difficulties in contacting their seniors for support, or may not feel comfortable doing so. Trainees may also feel like they would benefit from being more informed of the hospital situation, or added learning and educational opportunities from the shift. The aim of this project was to improve the out of hours support for the on-call SHOs - which we hope to have positive short (such as improving confidence and performance) - and longer-term impacts (improving retention in the deanery and specialty). 